\title{
Coulisses
}

Revue de théâtre

\section{John Webster, La Duchesse de Malfi [The Tragedy of the Duchesse of Malfy]}

Mise en scène d'Anne-Laure Liégeois au Nouveau Théâtre de Besançon, mars 2011

\section{David Ball}

\section{(2) OpenEdition}

\section{Journals}

Édition électronique

URL : https://journals.openedition.org/coulisses/428

DOI : $10.4000 /$ coulisses. 428

ISSN : 2546-9460

\section{Éditeur}

Presses universitaires de Franche-Comté

\section{Édition imprimée}

Date de publication : 31 décembre 2011

Pagination : 193-197

ISBN : 978-2-84867-404-9

ISSN : $1150-594 \mathrm{X}$

\section{Référence électronique}

David Ball, " John Webster, La Duchesse de Malfi [The Tragedy of the Duchesse of Malfy] », Coulisses [En ligne], 43 | Automne 2011, mis en ligne le 30 novembre 2016, consulté le 29 décembre 2022. URL http://journals.openedition.org/coulisses/428 ; DOI : https://doi.org/10.4000/coulisses.428 


\section{John Webster, La Duchesse de Malfi [The Tragedy of the Duchesse of Malfy]}

Mise en scène d'Anne-Laure Liégeois au Nouveau Théâtre de Besançon, mars 2011

David Ball

\section{RÉFÉRENCE}

John Webster, La Duchesse de Malfi [The Tragedy of the Duchesse of Malfy]

Mise en scène d'Anne-Laure Liégeois au Nouveau Théâtre de Besançon, mars 2011

$1 \quad$ La Duchesse de Malfi est sans doute la plus grande tragédie du théâtre élisabéthain, à part, bien entendu, presque toutes celles de Shakespeare. J'étais sur le point de dire : la plus grande pièce toutes catégories confondues, mais cela serait oublier les quatre ou cinq meilleures pièces de Ben Jonson, surtout Volpone et L'Alchimiste, dont la comédie satirique féroce comporte un élément tragique, tellement la cupidité des dupes est aveugle et l'énergie immense de ceux qui les trompent est dévoyée. Dans La Duchesse de Malfi tout le monde trompe ou espère tromper mais se trouve trompé à son tour.

Résumons l'intrigue, qui dans ses grandes lignes n'est pas trop compliquée. La Duchesse, jeune veuve, épouse en deuxièmes noces son intendant, Antonio, homme noble d'esprit mais pas de naissance. Un mariage qu'elle cache donc, par peur de la réaction violente de ses deux frères, Ferdinand, le duc de Calabre, et le Cardinal, trop fiers de leur sang noble. Bosola, l'espion du Cardinal, découvre d'abord les grossesses de la Duchesse, et puis c'est la Duchesse elle-même qui lui révèle naïvement l'identité du père, informations qu'il transmet à ses maîtres, qui décident de faire assassiner non seulement les deux fautifs mais aussi leurs trois enfants. Mais en voyant le corps étranglé au visage toujours beau de sa sœur jumelle, Ferdinand, pris de remords, tombe dans la folie, et le Cardinal, cherchant à terminer et à étouffer l'affaire en faisant assassiner tous ceux qui pourraient le gêner ou le trahir, trouve lui aussi la mort avec eux dans une confusion désespérée et meurtrière. Bosola tue Antonio par méprise et 
puis, volontairement, le Cardinal et le duc Ferdinand. Mais qui tue Bosola? Ferdinand, qui le blesse à mort, avant que Bosola ne le tue.

3 Je me ravise : même le résumé le plus sommaire est assez compliqué ! Retenons donc les mots clés de trahison et de vengeance. Ajoutons à cela l'impuissance, l'impuissance d'abord de l'innocence à résister aux forces déchaînées du mal, mais aussi celle de ces mêmes forces, incapables de maittriser toutes les conséquences de leurs gestes.

4 Au mal absolu des deux frères s'oppose l'innocence de la Duchesse, innocence qui est à la fois vertu et naïveté. Et entre ces deux extrêmes se situe l'ambiguïté de Bosola, malcontent mélancolique, l'agent et la victime d'une société déjà instable, où les vieilles valeurs féodales d'une hiérarchie sociale et morale se perdent dans l'individualisme du chacun pour soi. «Je me prendrai pour seul modèle » dit-il vers la fin, en rejetant et la grandeur d'âme de la Duchesse et d'Antonio et la bassesse maléfique de Ferdinand et du Cardinal.

5 Le malcontent est un personnage typique du théâtre de l'époque : un homme déclassé ou déguisé, marginal, qui se fait censeur et satiriste, et, par ses extravagances mêmes, se trouve aussi libre qu'un clown ou qu'un fou de critiquer tout le monde. Il y a une pièce de John Marston qui s'appelle justement Le Malcontent (The Malcontent, 1604), pour laquelle Webster écrivit une scène d'introduction quand elle fut reprise par les Comédiens du roi, la compagnie de Shakespeare et de Webster, une dizaine d'années avant La Duchesse de Malfi.

6 Le malcontent est différent du machiavel. Ce dernier, d'une vraie méchanceté, cherche à détruire par l'intrigue quiconque le gêne dans la réalisation de ses ambitions. L'Iago et l'Edmund de Shakespeare, tout comme le Juif de Malte de Marlowe, sont des machiavels, qui annoncent directement aux spectateurs la méchanceté qu'ils cachent aux autres personnages. Bosola, peut-on dire, est un mélange de malcontent et de machiavel. Il condamne ceux qu'il trouve pires que lui et dénonce les vices et les hypocrisies qu'il voit dans le monde mais il est sans scrupules lorsqu'il s'agit de défendre ses propres intérêts. "Ah! le vil métier d'espion!» s'écrie-t-il, en toute connaissance de cause, mais en se justifiant comme il peut : «Bah! tous les métiers du monde se valent! Ce qui compte, c'est le gain ou la gloire qu'on tire. » (acte III, sc. II)

7 Toutes les citations sont de la traduction de Gisèle Venet de 1992 (Belles Lettres), qui manque, malheureusement, à notre avis, de limpidité et de fidélité. La traduction de la mise en scène, par Anne-Laure Liégeois et Nigel Gearing, n'est pas tout à fait la même mais elle aurait pu être sans doute un peu plus claire et plus exacte.

Dans son «Introduction », la même Gisèle Venet emploie, pourtant, une expression juste à propos de Bosola : «l'ange exterminateur, le justicier sans justice, l'ange aveuglé par les valeurs du monde». C'est lui le responsable principal de l'hécatombe des personnages, avec laquelle se termine d'ailleurs la plupart des tragédies du théâtre élisabéthain, hécatombe où coupables et innocents trouvent ensemble une mort violente, comme si la mort méritée des seuls coupables n'aurait pas été suffisamment forte dans le tragique. Il est à remarquer, cependant, que Shakespeare, après Hamlet, la première de ses grandes tragédies, a tendance à éviter les hécatombes, où à la fin du dernier acte la scène est jonchée de corps. Même à la fin du Roi Lear, peut-être la plus noire, la plus terrible de toutes les tragédies, la plupart des morts ont lieu hors scène. Et plus loin encore, dans la pratique de la tragi-comédie de ses successeurs les plus en 
vue du théâtre londonien, Beaumont et Fletcher, ce sont même l'élégance et la douceur des mœurs qui commencent à régler l'évolution de l'intrigue.

Mais dans les morts violentes qui, à l'époque, se produisaient au théâtre public devant les yeux grands ouverts des spectateurs tout proches du plateau, il y avait, bien sûr, un élément de spectacle populaire. Une telle furie sanguinaire, pourtant, qui outrait les esprits néoclassiques du dix-huitième siècle, risque aujourd'hui de tout simplement nous faire rire. Et la metteure en scène a ici anticipé cette réaction, en faisant jouer Ferdinand et Bosola avec les poches de liquide rouge qu'ils portent sous leurs chemises pour les derniers coups de poignard. La furie sanguinaire se réduit ainsi à une farce d'enfants qui jouent avec la peinture mais l'épouvante de la mort se retrouve dans le positionnement pervers des corps enfin immobiles.

Pour infliger une torture morale à sa sœur, Ferdinand fait mettre sur scène devant elle des effigies en cire qui représentent, derrière un voile transparent, l'intendant Antonio et leurs enfants, morts, créant non seulement un effet de grand guignol populaire mais aussi une espèce de memento mori typique de l'iconographie baroque du $\mathrm{XvII}^{\mathrm{e}}$ siècle. Dans la mise en scène d'Anne-Laure Liégeois, c'est un Antonio nu qui passe rapidement au fond de la scène sur un chariot. Mais pour la mise à mort de la Duchesse on met sur le plateau un squelette et plusieurs rapaces empaillés. Il est clair que l'iconographie baroque, chère à la piété de la Contre-réforme catholique, touchait aussi des pays protestants. Et Webster, plus que d'autres, semble désireux de nous rappeler que sous la peau la plus belle, la plus lumineuse, se trouve toujours le crâne vide et ricanant. L'idée de la metteure en scène n'est pas ici très différente en soi mais beaucoup plus voyante au théâtre : que sous les vêtements les plus élaborés se trouve toujours le corps $\mathrm{nu}$, vulnérable et attendrissant.

11 Un aspect plus évidemment protestant du théâtre élisabéthain est la joie que semblent avoir eue ses auteurs à présenter des grands ecclésiastiques italiens comme cruels et corrompus. Le personnage du Cardinal dans la pièce de Webster est typique de cette vision à la fois xénophobe et sectaire. Il se débarrasse de sa maîtresse, une femme mariée, par une méthode qui en dit long sur les conceptions populaires des mœurs italiennes de l'époque : il lui fait embrasser un livre, sans doute une bible, dont la couverture est empoisonnée ! Et cet homme a failli devenir pape ! « Il aurait dû être fait Pape, dit de lui Antonio au début de la pièce, mais au lieu de s'y préparer avec l'humilité de l'Église primitive, il a préféré soudoyer son entourage par une prodigalité impudente, à croire qu'il cherchait à l'emporter à l'insu du ciel. »

Lors d'une autre épreuve morale infligée à sa sœur, Ferdinand fait jouer à côté de sa chambre des fous, qui parlent comme des malcontents mais de façon plus décousue, et Bosola, déguisé, joue à être fou avec eux de la même façon. Mais quand, un peu plus tard, Ferdinand tombe lui-même dans la folie, il se croit transformé en loup et obligé ainsi de déterrer des corps des cimetières. Ces deux variétés de la folie dans la pièce, la mécontente et la macabre, deviennent, dans la mise en scène, plus farfelues, comme le comique d'un fou qui traverse le plateau un ballon attaché à une ficelle au-dessus de la tête. On se moquait des fous aux XVI ${ }^{\mathrm{e}}$ et XVII ${ }^{\mathrm{e}}$ siècles; on les battait, pour en chasser les démons, mais on les tolérait parfois aussi, dans l'espoir d'en tirer une inspiration, divine ou autre. Et de toute manière, avec sa vocifération et son coq-à-l'âne, la folie fait toujours du bon théâtre!

De l'auteur, John Webster, nous savons très peu de choses. On suppose qu'il est né autour de 1580 et mort vers 1630 mais aucun document ne l'atteste. De ses activités 
littéraires, poétiques et théâtrales, nous avons quelques traces mais ce qui nous reste de précieux de lui ce sont ses deux tragédies, Le Démon blanc et La Duchesse de Malfi, écrites dans les années 1612,1614, et toutes deux remarquables pour leur goût du macabre, leur fascination à l'égard d'une Italie chaotique et corrompue, et leurs deux héroïnes, la Duchesse et Vittoria Corombona, femmes d'esprit et de courage. Vittoria, le démon blanc, moins vertueuse donc que la Duchesse, est une femme mariée détruite par son amour pour un grand homme impétueux prêt à tuer pour pouvoir l'épouser.

Les rôles principaux de la Duchesse et de Bosola sont très bien assurés dans cette mise en scène par Valérie Schwarcz et Olivier Constant. On n'a pas lésiné sur le nombre d'acteurs employés. Il y en a même suffisamment pour faire un entourage de quatre courtisans pour Ferdinand, la Duchesse ou le Cardinal, entourage d'une flagornerie, comme il se doit, grotesque et dérisoire. Les autres rôles de personnages nobles, nobles de sang ou d'esprit, Ferdinand (Olivier Dutilloy), le Cardinal (Nils Öhlund) et Antonio (Sébastien Bravard), sont joués pour la plupart avec passion et sérieux, même si la passion s'exclame parfois un peu trop fort. Ferdinand est certes un personnage qui maitrise mal sa colère mais s'il crie trop fort certains des spectateurs auront des difficultés à le comprendre.

Le décor, par contre, est d'une grande sobriété, sans changement, sombre et austère : une immense salle aux murs vides et presque noirs dont les portes étroites sont cachées dans les panneaux. Au fond il $\mathrm{y}$ a une arrière scène, un peu comme au théâtre élisabéthain, qui représente la chambre des époux ou un ailleurs, un dehors, selon les déplacements des personnages. Les lumières sont atténuées, avec parfois des effets de brillance qui frappent comme un éclair. Et la musique est lente, forte, avec une dominance évidente du violoncelle.

Les costumes aussi sont pour la plupart sombres, du noir et blanc, mais, prêtés par la Comédie-Française, ils sont somptueux, dans le style Renaissance italienne des portraits d'homme et de femme de Titien. Le Cardinal porte une soutane d'un rouge sang éclatant, et la Duchesse aussi s'habille en rouge après le départ de son mari. Est-ce la couleur d'une victime sacrificielle, de la putain de l'imagination de ses frères, ou de la femme mûre, mère de famille... ?

17 Un aspect remarquable de cette pièce est l'insistance de la Duchesse, mais aussi de Bosola, qui le dit sans doute sincèrement, que la vraie noblesse n'est pas dans la naissance mais dans l'âme et l'esprit, avec, comme corollaire, que le mariage doit être le libre choix des deux époux. Ce sont là deux lieux communs, peut-être, mais leur répétition donne à la pièce, autrement très évidemment d'un autre âge, une modernité surprenante et bienvenue, que la mise en scène a su mettre en valeur. 
INDEX

oeuvrecitee Duchesse de Malfi (La)

Mots-clés : mise en scène

Keywords : mise-en-scène

Palabras claves : puesta en escena

Palavras-chave : cenografia 